\title{
On the Ellipsoid and Plane Intersection Equation
}

\author{
Peter Paul Klein \\ Computing Center, University of Technology Clausthal, Clausthal-Zellerfeld, Germany \\ Email: klein@rz.tu-clausthal.de
}

Received August 3, 2012; revised September 10, 2012; accepted September 17, 2012

\begin{abstract}
It is well known that the line of intersection of an ellipsoid and a plane is an ellipse. In this note simple formulas for the semi-axes and the center of the ellipse are given, involving only the semi-axes of the ellipsoid, the componentes of the unit normal vector of the plane and the distance of the plane from the center of coordinates. This topic is relatively common to study, but, as indicated in [1], a closed form solution to the general problem is actually very difficult to derive. This is attemped here. As applications problems are treated, which were posed in the internet $[1,2]$, pertaining to satellite orbits in space and to planning radio-therapy treatment of eyes.
\end{abstract}

Keywords: Ellipsoid and Plane Intersection; Identity of Lagrange; Grassmann Expansion Theorem

\section{Introduction}

Let an ellipsoid be given with the three positive semiaxes $a, b, c$

$$
\frac{x_{1}^{2}}{a^{2}}+\frac{x_{2}^{2}}{b^{2}}+\frac{x_{3}^{2}}{c^{2}}=1
$$

and a plane with the unit normal vector

$$
\boldsymbol{n}=\left(n_{1}, n_{2}, n_{3}\right)^{\mathrm{T}}
$$

which contains an interior point $\boldsymbol{q}=\left(q_{1}, q_{2}, q_{3}\right)^{\mathrm{T}}$ of the ellipsoid. A plane spanned by vectors $\boldsymbol{r}=\left(r_{1}, r_{2}, r_{3}\right)^{\mathrm{T}}$, $\boldsymbol{s}=\left(s_{1}, s_{2}, s_{3}\right)^{\mathrm{T}}$ and containing the point $\boldsymbol{q}$ is described in parametric form by

$$
\begin{aligned}
& \boldsymbol{x}=\boldsymbol{q}+\text { tr }+ \text { us } \\
& \text { with } \quad \boldsymbol{x}=\left(x_{1}, x_{2}, x_{3}\right)^{\mathrm{T}} .
\end{aligned}
$$

Inserting the components of $\boldsymbol{x}$ into the equation of the ellipsoid (1) leads to the line of intersection as a quadratic form in the variables $t$ and $u$. Let the scalar product in $R^{3}$ for two vectors $\boldsymbol{v}=\left(v_{1}, v_{2}, v_{3}\right)^{\mathrm{T}}$ and $\boldsymbol{w}=\left(w_{1}, w_{2}, w_{3}\right)^{\mathrm{T}}$ be denoted by

$$
(\boldsymbol{v}, \boldsymbol{w})=v_{1} w_{1}+v_{2} w_{2}+v_{3} w_{3} .
$$

With the diagonal matrix

$$
D_{1}=\operatorname{diag}\left(\frac{1}{a}, \frac{1}{b}, \frac{1}{c}\right)
$$

the line of intersection has the form:

$$
\begin{aligned}
& (t, u)\left(\begin{array}{ll}
\left(D_{1} \boldsymbol{r}, D_{1} \boldsymbol{r}\right) & \left(D_{1} \boldsymbol{r}, D_{1} \boldsymbol{s}\right) \\
\left(D_{1} \boldsymbol{r}, D_{1} \boldsymbol{s}\right) & \left(D_{1} \boldsymbol{s}, D_{1} \boldsymbol{s}\right)
\end{array}\right)\left(\begin{array}{l}
t \\
u
\end{array}\right) \\
& +2\left(\left(D_{1} \boldsymbol{q}, D_{1} \boldsymbol{r}\right),\left(D_{1} \boldsymbol{q}, D_{1} \boldsymbol{s}\right)\right)\left(\begin{array}{l}
t \\
u
\end{array}\right) \\
& =1-\left(D_{1} \mathbf{q}, D_{1} \boldsymbol{q}\right) .
\end{aligned}
$$

As $\boldsymbol{q}$ is an interior point of the ellipsoid the righthand side of Equation (2) is positive. The $2 \times 2$ matrix in Equation (3) is a Gram matrix. If the vectors $D_{1} \boldsymbol{r}$ and $D_{1} s$ are linearly independent, this is equivalent with the linear independence of the vectors $\boldsymbol{r}$ and $\boldsymbol{s}$, the matrix in (3) is positive definite and the line of intersection is an ellipse. In [3] a generalization from three to p-dimensional space is discussed.

Let $\boldsymbol{r}$ and $\boldsymbol{s}$ be unit vectors orthogonal to the unit normal vector $\boldsymbol{n}$ of the plane

$$
\begin{aligned}
& (\boldsymbol{r}, \boldsymbol{r})=r_{1}^{2}+r_{2}^{2}+r_{3}^{2}=1 \\
& (\boldsymbol{n}, \boldsymbol{r})=n_{1} r_{1}+n_{2} r_{2}+n_{3} r_{3}=0 \\
& (\boldsymbol{s}, \boldsymbol{s})=s_{1}^{2}+s_{2}^{2}+s_{3}^{2}=1 \\
& (\boldsymbol{n}, \boldsymbol{s})=n_{1} s_{1}+n_{2} s_{2}+n_{3} s_{3}=0
\end{aligned}
$$

and orthogonal to eachother

$$
(\boldsymbol{r}, \boldsymbol{s})=r_{1} s_{1}+r_{2} s_{2}+r_{3} s_{3}=0 .
$$

Furthermore vectors $\boldsymbol{r}$ and $\boldsymbol{s}$ may be chosen such that

$$
\left(D_{1} \boldsymbol{r}, D_{1} \boldsymbol{s}\right)=\frac{r_{1} S_{1}}{a^{2}}+\frac{r_{2} S_{2}}{b^{2}}+\frac{r_{3} S_{3}}{c^{2}}=0
$$


holds. This will be shown in the next section. Condition (7) ensures that the $2 \times 2$ matrix in (3) has diagonal form. Then the line of intersection reduces to an ellipse in translational form

$$
\frac{\left(t-t_{0}\right)^{2}}{A^{2}}+\frac{\left(u-u_{0}\right)^{2}}{B^{2}}=1
$$

with the center $\left(t_{0}, u_{0}\right)$

$$
t_{0}=-\frac{\left(D_{1} \boldsymbol{q}, D_{1} \boldsymbol{r}\right)}{\left(D_{1} \boldsymbol{r}, D_{1} \boldsymbol{r}\right)} \quad \text { and } \quad u_{0}=-\frac{\left(D_{1} \boldsymbol{q}, D_{1} \boldsymbol{s}\right)}{\left(D_{1} \boldsymbol{s}, D_{1} \boldsymbol{s}\right)}
$$

and the semi-axes

$$
A=\sqrt{\frac{1-d}{\left(D_{1} \boldsymbol{r}, D_{1} \boldsymbol{r}\right)}} \quad \text { and } \quad B=\sqrt{\frac{1-d}{\left(D_{1} \boldsymbol{s}, D_{1} s\right)}},
$$

where

$$
d=\left(D_{1} \boldsymbol{q}, D_{1} \boldsymbol{q}\right)-\frac{\left(D_{1} \boldsymbol{q}, D_{1} \boldsymbol{r}\right)^{2}}{\left(D_{1} \boldsymbol{r}, D_{1} \boldsymbol{r}\right)}-\frac{\left(D_{1} \boldsymbol{q}, D_{1} \boldsymbol{s}\right)^{2}}{\left(D_{1} \boldsymbol{s}, D_{1} \boldsymbol{s}\right)} .
$$

In order to show that the semi-axes (10) are independent of the choice of $\boldsymbol{q}$ this vector may be decomposed orthogonally with respect to $\boldsymbol{n}$ :

$$
\boldsymbol{q}=\boldsymbol{\kappa} \boldsymbol{n}+\mu \boldsymbol{r}+\nu \boldsymbol{s} \quad \text { with } \quad \kappa=(\boldsymbol{q}, \boldsymbol{n})
$$

where $\kappa$ is the distance of plane (2) from the origin. Substituting $\boldsymbol{q}$ into (11) one obtains employing (4), (5), (6) and (7)

$$
d=\kappa^{2}\left(\left(D_{1} \boldsymbol{n}, D_{1} \boldsymbol{n}\right)-\frac{\left(D_{1} \boldsymbol{n}, D_{1} \boldsymbol{r}\right)^{2}}{\left(D_{1} \boldsymbol{r}, D_{1} \boldsymbol{r}\right)}-\frac{\left(D_{1} \boldsymbol{n}, D_{1} \boldsymbol{s}\right)^{2}}{\left(D_{1} \boldsymbol{s}, D_{1} \boldsymbol{s}\right)}\right) .
$$

The following rules of computation for the cross product in $R^{3}$ ([4, p.147]) will be applied later on repeatedly. For vectors $\boldsymbol{x}, \boldsymbol{y}, \mathbf{z}, \boldsymbol{w}$ of $R^{3}$ the identity of Lagrange holds

$$
(x \times y, z \times w)=(x, z)(y, w)-(y, z)(x, w)
$$

and the Grassmann expansion theorem for the double cross product

$$
x \times(y \times z)=(x, z) y-(x, y) z .
$$

\section{Construction of Vectors $r$ and $s$}

Let $\boldsymbol{r}$ be a unit vector orthogonal to the unit normal vector $\boldsymbol{n}$ of the plane, so that Equations (4) hold. A suitable vector $s$ is obtained as a cross product

$$
\boldsymbol{s}=\boldsymbol{n} \times \boldsymbol{r} .
$$

Then Equations (5) and (6) are fulfilled: $s$ is a unit vector, as can be shown by the identity of Lagrange (14), utilising $(\boldsymbol{n}, \boldsymbol{n})=1,(\boldsymbol{r}, \boldsymbol{r})=1$ and $(\boldsymbol{n}, \boldsymbol{r})=0$ :

$$
(\boldsymbol{s}, \boldsymbol{s})=(\boldsymbol{n} \times \boldsymbol{r}, \boldsymbol{n} \times \boldsymbol{r})=(\boldsymbol{n}, \boldsymbol{n})(\boldsymbol{r}, \boldsymbol{r})-(\boldsymbol{n}, \boldsymbol{r})^{2}=1 .
$$

Furthermore one obtains according to the rules apply- ing to the spar product:

$$
\begin{gathered}
(\boldsymbol{n}, \boldsymbol{s})=(\boldsymbol{n}, \boldsymbol{n} \times \boldsymbol{r})=\operatorname{det}(\boldsymbol{n}, \boldsymbol{n}, \boldsymbol{r})=0, \\
(\boldsymbol{r}, \boldsymbol{s})=(\boldsymbol{r}, \boldsymbol{n} \times \boldsymbol{r})=\operatorname{det}(\boldsymbol{r}, \boldsymbol{n}, \boldsymbol{r})=0 .
\end{gathered}
$$

In case Equation (7) is not fulfilled for the initially chosen vectors $\boldsymbol{r}$ and $\boldsymbol{s}$, i.e. $\left(D_{1} \boldsymbol{r}, D_{1} \boldsymbol{s}\right) \neq 0$, the following transformation may be performed with $\omega \in[-\pi, \pi)$

$$
\begin{aligned}
& \tilde{\boldsymbol{r}}=\cos \omega \boldsymbol{r}+\sin \omega \mathbf{s}, \\
& \tilde{\boldsymbol{s}}=-\sin \omega \mathbf{r}+\cos \omega \mathbf{s} .
\end{aligned}
$$

The transformed vectors $\tilde{\boldsymbol{r}}$ and $\tilde{\boldsymbol{s}}$ satisfy the following conditions: $(\tilde{\boldsymbol{r}}, \tilde{\boldsymbol{r}})=1,(\boldsymbol{n}, \tilde{\boldsymbol{r}})=0$ and $\tilde{\boldsymbol{s}}=\boldsymbol{n} \times \tilde{\boldsymbol{r}}$, which imply conditions (4)-(6). The expression

$$
\begin{aligned}
\left(D_{1} \tilde{\boldsymbol{r}}, D_{1} \tilde{\boldsymbol{s}}\right) & =\left(\left(D_{1} \boldsymbol{s}, D_{1} \boldsymbol{s}\right)-\left(D_{1} \boldsymbol{r}, D_{1} \boldsymbol{r}\right)\right) \frac{1}{2} \sin 2 \omega \\
& +\left(D_{1} \boldsymbol{r}, D_{1} \boldsymbol{s}\right) \cos 2 \omega
\end{aligned}
$$

becomes zero, when choosing $\omega$ such that

$$
\frac{\left(D_{1} \boldsymbol{r}, D_{1} \boldsymbol{r}\right)-\left(D_{1} \boldsymbol{s}, D_{1} \boldsymbol{s}\right)}{2\left(D_{1} \boldsymbol{r}, D_{1} \boldsymbol{s}\right)}=\cot 2 \omega
$$

holds. This can be reformulated, in case

$$
\left(D_{1} \boldsymbol{r}, D_{1} \boldsymbol{r}\right)-\left(D_{1} \boldsymbol{s}, D_{1} \boldsymbol{s}\right) \neq 0
$$

to

$$
\omega=\frac{1}{2} \arctan \left[\frac{2\left(D_{1} \boldsymbol{r}, D_{1} \boldsymbol{s}\right)}{\left(D_{1} \boldsymbol{r}, D_{1} \boldsymbol{r}\right)-\left(D_{1} \boldsymbol{s}, D_{1} \boldsymbol{s}\right)}\right] .
$$

If

$$
\left(D_{1} \boldsymbol{r}, D_{1} \boldsymbol{r}\right)-\left(D_{1} \boldsymbol{s}, D_{1} \boldsymbol{s}\right)=0
$$

holds, $\omega$ can be chosen $\pi / 4$, leading to $\sin (\pi / 4)=\cos (\pi / 4)=1 / \sqrt{2}$.

Corollary 1: For the unit vectors $\boldsymbol{r}$ and $\boldsymbol{n}$ orthogonal to each other and $\boldsymbol{s}=\boldsymbol{n} \times \boldsymbol{r}$ the following statement holds:

$$
r_{i}^{2}+s_{i}^{2}+n_{i}^{2}=1 \quad \text { for } \quad i=1,2,3 .
$$

Statement (17) follows by substituting the definition of $\boldsymbol{s}$ and utilising $(\boldsymbol{n}, \boldsymbol{n})=1,(\boldsymbol{r}, \boldsymbol{r})=1$ and $(\boldsymbol{n}, \boldsymbol{r})=0$. For $i=1$ one obtains for instance:

$$
\begin{aligned}
& r_{1}^{2}+s_{1}^{2}+n_{1}^{2} \\
= & r_{1}^{2}+\left(n_{2} r_{3}-r_{2} n_{3}\right)^{2}+n_{1}^{2} \\
= & r_{1}^{2}+n_{2}^{2} r_{3}^{2}-2 n_{2} r_{3} n_{3} r_{2}+r_{2}^{2} n_{3}^{2}+1-n_{2}^{2}-n_{3}^{2} \\
= & r_{1}^{2}-n_{2}^{2}\left(1-r_{3}^{2}\right)-2 n_{2} r_{2} n_{3} r_{3}-n_{3}^{2}\left(1-r_{2}^{2}\right)+1 \\
= & r_{1}^{2}-n_{2}^{2}\left(r_{1}^{2}+r_{2}^{2}\right)-2 n_{2} r_{2} n_{3} r_{3}-n_{3}^{2}\left(r_{1}^{2}+r_{3}^{2}\right)+1 \\
= & r_{1}^{2}\left(1-n_{2}^{2}-n_{3}^{2}\right)-\left(n_{2} r_{2}+n_{3} r_{3}\right)^{2}+1 \\
= & r_{1}^{2} n_{1}^{2}-\left(-n_{1} r_{1}\right)^{2}+1=1 .
\end{aligned}
$$




\section{A Quadratic Equation}

Theorem 1: Let $\boldsymbol{n}$ be the unit normal vector of the plane and let vectors $\boldsymbol{r}$ and $\boldsymbol{s}$ satisfy $(\boldsymbol{r}, \boldsymbol{r})=1$, $(\boldsymbol{n}, \boldsymbol{r})=0, \boldsymbol{s}=\boldsymbol{n} \times \boldsymbol{r}$ and condition (7). Putting

$$
\begin{aligned}
& \beta_{1}=\left(D_{1} \boldsymbol{r}, D_{1} \boldsymbol{r}\right)=\frac{r_{1}^{2}}{a^{2}}+\frac{r_{2}^{2}}{b^{2}}+\frac{r_{3}^{2}}{c^{2}}, \\
& \beta_{2}=\left(D_{1} \boldsymbol{s}, D_{1} \boldsymbol{s}\right)=\frac{s_{1}^{2}}{a^{2}}+\frac{s_{2}^{2}}{b^{2}}+\frac{s_{3}^{2}}{c^{2}},
\end{aligned}
$$

$\beta_{1}$ and $\beta_{2}$ are solutions of the following quadratic equation:

$$
\begin{aligned}
& \beta^{2}-\left[n_{1}^{2}\left(\frac{1}{b^{2}}+\frac{1}{c^{2}}\right)+n_{2}^{2}\left(\frac{1}{a^{2}}+\frac{1}{c^{2}}\right)+n_{3}^{2}\left(\frac{1}{a^{2}}+\frac{1}{b^{2}}\right)\right] \beta \\
& +\frac{n_{1}^{2}}{b^{2} c^{2}}+\frac{n_{2}^{2}}{a^{2} c^{2}}+\frac{n_{3}^{2}}{a^{2} b^{2}}=0 .
\end{aligned}
$$

Proof: Utilising (17) one obtains:

$$
\begin{aligned}
\beta_{1}+\beta_{2} & =\frac{r_{1}^{2}+s_{1}^{2}}{a^{2}}+\frac{r_{2}^{2}+s_{2}^{2}}{b^{2}}+\frac{r_{3}^{2}+s_{3}^{2}}{c^{2}} \\
& =\frac{1-n_{1}^{2}}{a^{2}}+\frac{1-n_{2}^{2}}{b^{2}}+\frac{1-n_{3}^{2}}{c^{2}} \\
& =\frac{n_{2}^{2}+n_{3}^{2}}{a^{2}}+\frac{n_{1}^{2}+n_{3}^{2}}{b^{2}}+\frac{n_{1}^{2}+n_{2}^{2}}{c^{2}} \\
& =n_{1}^{2}\left(\frac{1}{b^{2}}+\frac{1}{c^{2}}\right)+n_{2}^{2}\left(\frac{1}{a^{2}}+\frac{1}{c^{2}}\right)+n_{3}^{2}\left(\frac{1}{a^{2}}+\frac{1}{b^{2}}\right) .
\end{aligned}
$$

Applying diagonality condition (7) and the identity of Lagrange (14) leads to:

$$
\begin{aligned}
\beta_{1} \beta_{2} & =\left(D_{1} \boldsymbol{r}, D_{1} \boldsymbol{r}\right)\left(D_{1} \boldsymbol{s}, D_{1} \boldsymbol{s}\right) \\
& =\left(D_{1} \boldsymbol{r}, D_{1} \boldsymbol{r}\right)\left(D_{1} \boldsymbol{s}, D_{1} \boldsymbol{s}\right)-\left(D_{1} \boldsymbol{r}, D_{1} \boldsymbol{s}\right)^{2} \\
& =\left(D_{1} \boldsymbol{r} \times D_{1} \boldsymbol{s}, D_{1} \boldsymbol{r} \times D_{1} \boldsymbol{s}\right) .
\end{aligned}
$$

For the cross product $D_{1} r \times D_{1} s$ one obtains:

$$
\begin{aligned}
D_{1} \boldsymbol{r} \times D_{1} \boldsymbol{s} & =\left|\begin{array}{ccc}
e_{1} & e_{2} & e_{3} \\
\frac{r_{1}}{a} & \frac{r_{2}}{b} & \frac{r_{3}}{c} \\
\frac{s_{1}}{a} & \frac{s_{2}}{b} & \frac{s_{3}}{c}
\end{array}\right|=\left(\begin{array}{c}
\frac{r_{2} s_{3}-s_{2} r_{3}}{b c} \\
\frac{s_{1} r_{3}-r_{1} s_{3}}{a c} \\
\frac{r_{1} s_{2}-s_{1} r_{2}}{a b}
\end{array}\right) \\
& =\tilde{D}_{1}(\boldsymbol{r} \times \boldsymbol{s}),
\end{aligned}
$$

with the diagonal matrix

$$
\tilde{D}_{1}=\operatorname{diag}\left(\frac{1}{b c}, \frac{1}{a c}, \frac{1}{a b}\right) .
$$

According to Grassmann's expansion theorem for the double cross product (15)

$$
\begin{aligned}
\tilde{D}_{1}(\boldsymbol{r} \times \boldsymbol{s}) & =\tilde{D}_{1}(\boldsymbol{r} \times(\boldsymbol{n} \times \boldsymbol{r})) \\
& =\tilde{D}_{1}((\boldsymbol{r}, \boldsymbol{r}) \boldsymbol{n}-(\boldsymbol{r}, \boldsymbol{n}) \boldsymbol{r})=\tilde{D}_{1} \boldsymbol{n},
\end{aligned}
$$

follows, since $(\boldsymbol{r}, \boldsymbol{r})=1$ and $(\boldsymbol{r}, \boldsymbol{n})=0$. Applying (20), (21), (23) one obtains:

$$
\beta_{1} \beta_{2}=\left(\tilde{D}_{1} \boldsymbol{n}, \tilde{D}_{1} \boldsymbol{n}\right)=\frac{n_{1}^{2}}{b^{2} c^{2}}+\frac{n_{2}^{2}}{a^{2} c^{2}}+\frac{n_{3}^{2}}{a^{2} b^{2}} .
$$

A quadratic equation equivalent to (19) is considered in [5].

Corollary 2: Under the assumptions of Theorem 1 the following three equations are valid:

$$
\begin{aligned}
& D_{1} \boldsymbol{r} \times D_{1} \boldsymbol{s}=\tilde{D}_{1}(\boldsymbol{r} \times \boldsymbol{s})=\tilde{D}_{1} \boldsymbol{n}, \\
& D_{1} \boldsymbol{n} \times D_{1} \boldsymbol{r}=\tilde{D}_{1}(\boldsymbol{n} \times \boldsymbol{r})=\tilde{D}_{1} \boldsymbol{s}, \\
& D_{1} \boldsymbol{s} \times D_{1} n=\tilde{D}_{1}(\boldsymbol{s} \times \boldsymbol{n})=\tilde{D}_{1} \boldsymbol{r} .
\end{aligned}
$$

The first of the three equations was verified in the proof of Theorem 1. The second and the third equation follow analogously.

\section{A Formular for $d$}

Theorem 2: Under the assumptions of Theorem 1 the expression for $d$ in (13) is given by:

$$
d=\frac{\kappa^{2}}{a^{2} n_{1}^{2}+b^{2} n_{2}^{2}+c^{2} n_{3}^{2}},
$$

where $\kappa$ is taken from (12).

Proof: The verification of (25) consists of three steps.

Step 1: Applying the identity of Lagrange (14) the following statements hold:

$$
\begin{aligned}
& \left(D_{1} \boldsymbol{n}, D_{1} \boldsymbol{n}\right)\left(D_{1} \boldsymbol{r}, D_{1} \boldsymbol{r}\right)-\left(D_{1} \boldsymbol{n}, D_{1} \boldsymbol{r}\right)^{2} \\
& =\left(D_{1} \boldsymbol{n} \times D_{1} \boldsymbol{r}, D_{1} \boldsymbol{n} \times D_{1} \boldsymbol{r}\right), \\
& \left(D_{1} \boldsymbol{n}, D_{1} \boldsymbol{n}\right)\left(D_{1} \boldsymbol{s}, D_{1} \boldsymbol{s}\right)-\left(D_{1} \boldsymbol{n}, D_{1} \boldsymbol{s}\right)^{2} \\
& =\left(D_{1} \boldsymbol{n} \times D_{1} \boldsymbol{s}, D_{1} \boldsymbol{n} \times D_{1} \boldsymbol{s}\right) .
\end{aligned}
$$

With Corollary 2 and the diagonal matrix

$$
D=\operatorname{diag}(a, b, c)
$$

one obtains:

$$
\begin{aligned}
& \left(D_{1} \boldsymbol{n} \times D_{1} \boldsymbol{r}, D_{1} \boldsymbol{n} \times D_{1} \boldsymbol{r}\right)=\left(\tilde{D}_{1} \boldsymbol{s}, \tilde{D}_{1} \boldsymbol{s}\right)=\frac{(D \boldsymbol{s}, D \boldsymbol{s})}{a^{2} b^{2} c^{2}}, \\
& \left(D_{1} \boldsymbol{n} \times D_{1} \boldsymbol{s}, D_{1} \boldsymbol{n} \times D_{1} \boldsymbol{s}\right)=\left(\tilde{D}_{1} \boldsymbol{r}, \tilde{D}_{1} \boldsymbol{r}\right)=\frac{(D \boldsymbol{r}, D \boldsymbol{r})}{a^{2} b^{2} c^{2}}
\end{aligned}
$$

and it follows by substituting (28) into (26)

$$
\begin{aligned}
& \left(D_{1} \boldsymbol{n}, D_{1} \boldsymbol{r}\right)^{2}=\left(D_{1} \boldsymbol{n}, D_{1} \boldsymbol{n}\right)\left(D_{1} \boldsymbol{r}, D_{1} \boldsymbol{r}\right)-\frac{(D \boldsymbol{s}, D \boldsymbol{s})}{a^{2} b^{2} c^{2}}, \\
& \left(D_{1} \boldsymbol{n}, D_{1} \boldsymbol{s}\right)^{2}=\left(D_{1} \boldsymbol{n}, D_{1} \boldsymbol{n}\right)\left(D_{1} \boldsymbol{s}, D_{1} \boldsymbol{s}\right)-\frac{(D \boldsymbol{r}, D \boldsymbol{r})}{a^{2} b^{2} c^{2}} .
\end{aligned}
$$


Introducing expressions

$$
\begin{aligned}
& \gamma_{1}=(D \boldsymbol{r}, D \boldsymbol{r})=a^{2} r_{1}^{2}+b^{2} r_{2}^{2}+c^{2} r_{3}^{2}, \\
& \gamma_{2}=(D \boldsymbol{s}, D \boldsymbol{s})=a^{2} s_{1}^{2}+b^{2} s_{2}^{2}+c^{2} s_{3}^{2},
\end{aligned}
$$

one obtains from (29) using (18) and (30)

$$
\begin{aligned}
& \left(D_{1} \boldsymbol{n}, D_{1} \boldsymbol{r}\right)^{2}=\left(D_{1} \boldsymbol{n}, D_{1} \boldsymbol{n}\right) \beta_{1}-\frac{\gamma_{2}}{a^{2} b^{2} c^{2}}, \\
& \left(D_{1} \boldsymbol{n}, D_{1} \boldsymbol{s}\right)^{2}=\left(D_{1} \boldsymbol{n}, D_{1} \boldsymbol{n}\right) \beta_{2}-\frac{\gamma_{1}}{a^{2} b^{2} c^{2}} .
\end{aligned}
$$

Combining both Equations (31) leads to

$$
\begin{aligned}
& \frac{\left(D_{1} \boldsymbol{n}, D_{1} \boldsymbol{r}\right)^{2}}{\left(D_{1} \boldsymbol{r}, D_{1} \boldsymbol{r}\right)}+\frac{\left(D_{1} \boldsymbol{n}, D_{1} \boldsymbol{s}\right)^{2}}{\left(D_{1} \boldsymbol{s}, D_{1} \boldsymbol{s}\right)} \\
& =2\left(D_{1} \boldsymbol{n}, D_{1} \boldsymbol{n}\right)-\frac{\gamma_{1} \beta_{1}+\gamma_{2} \beta_{2}}{a^{2} b^{2} c^{2} \beta_{1} \beta_{2}} .
\end{aligned}
$$

Step 2: Analogously to the verification of (24) the application of the identity of Lagrange (14) yields:

$$
\begin{aligned}
\gamma_{1} \gamma_{2}= & (D \boldsymbol{r}, D \boldsymbol{r})(D \boldsymbol{s}, D \boldsymbol{s})=(D \boldsymbol{r}, D \boldsymbol{r})(D \mathbf{s}, D \boldsymbol{s}) \\
& -(D \boldsymbol{r}, D \boldsymbol{s})^{2}+(D \boldsymbol{r}, D \boldsymbol{s})^{2} \\
= & (D \boldsymbol{r} \times D \boldsymbol{s}, D \boldsymbol{r} \times D \boldsymbol{s})+(D \boldsymbol{r}, D \boldsymbol{s})^{2} .
\end{aligned}
$$

With the diagonal matrix $\tilde{D}=\operatorname{diag}(b c, a c, a b)$ for the cross product $\operatorname{Dr} \times D s$ holds:

$$
D \boldsymbol{r} \times D \boldsymbol{s}=\tilde{D}(\boldsymbol{r} \times \boldsymbol{s})=\tilde{D} \boldsymbol{n} .
$$

Therefore one obtains

$$
\gamma_{1} \gamma_{2}=(\tilde{D} \boldsymbol{n}, \tilde{D} \boldsymbol{n})+(D \boldsymbol{r}, D \boldsymbol{s})^{2}
$$

or

$$
\gamma_{1} \gamma_{2}=a^{2} b^{2} c^{2}\left(D_{1} \boldsymbol{n}, D_{1} \boldsymbol{n}\right)+(D \boldsymbol{r}, D \boldsymbol{s})^{2} .
$$

In contrast to the verification of (24), where diagonality condition (7) holds, the analogous expression $(D r, D s)$ in (33) need not be zero.

Step 3: Applying the identity of Lagrange (14) again leads to

$$
\begin{aligned}
& \left(D_{1} \boldsymbol{n} \times D_{1} \boldsymbol{r}, D_{1} \boldsymbol{n} \times D_{1} \boldsymbol{s}\right) \\
& =\left(D_{1} \boldsymbol{n}, D_{1} \boldsymbol{n}\right)\left(D_{1} \boldsymbol{r}, D_{1} \boldsymbol{s}\right)-\left(D_{1} \boldsymbol{n}, D_{1} \boldsymbol{s}\right)\left(D_{1} \boldsymbol{r}, D_{1} \boldsymbol{n}\right) .
\end{aligned}
$$

Substituting the involved cross products according to Corollary 2 and considering diagonality condition (7) one obtains

$$
\left(\tilde{D}_{1} \boldsymbol{s}, \tilde{D}_{1} \boldsymbol{r}\right)=\left(D_{1} \boldsymbol{n}, D_{1} \boldsymbol{s}\right)\left(D_{1} \boldsymbol{n}, D_{1} \boldsymbol{r}\right)
$$

or

$$
\frac{(D \boldsymbol{s}, D \boldsymbol{r})}{a^{2} b^{2} c^{2}}=\left(D_{1} \boldsymbol{n}, D_{1} \boldsymbol{s}\right)\left(D_{1} \boldsymbol{n}, D_{1} \boldsymbol{r}\right) .
$$

Squaring both sides of (34) and substituting the expressions from (31) leads to:

$$
\begin{aligned}
& (D \boldsymbol{s}, D \boldsymbol{r})^{2} \\
& =\left(a^{2} b^{2} c^{2}\left(D_{1} \boldsymbol{n}, D_{1} \boldsymbol{n}\right) \beta_{2}-\gamma_{1}\right)\left(a^{2} b^{2} c^{2}\left(D_{1} \boldsymbol{n}, D_{1} \boldsymbol{n}\right) \beta_{1}-\gamma_{2}\right) \\
& =a^{4} b^{4} c^{4}\left(D_{1} \boldsymbol{n}, D_{1} \boldsymbol{n}\right)^{2} \beta_{1} \beta_{2} \\
& -\left(\gamma_{1} \beta_{1}+\gamma_{2} \beta_{2}\right) a^{2} b^{2} c^{2}\left(D_{1} \boldsymbol{n}, D_{1} \boldsymbol{n}\right)+\gamma_{1} \gamma_{2} .
\end{aligned}
$$

Substitution of (33) results in equation

$$
\begin{aligned}
& a^{2} b^{2} c^{2}\left(D_{1} \boldsymbol{n}, D_{1} \boldsymbol{n}\right) \\
& \cdot\left[a^{2} b^{2} c^{2}\left(D_{1} \boldsymbol{n}, D_{1} \boldsymbol{n}\right) \beta_{1} \beta_{2}-\left(\gamma_{1} \beta_{1}+\gamma_{2} \beta_{2}\right)+1\right] \\
& =0,
\end{aligned}
$$

or

$$
\gamma_{1} \beta_{1}+\gamma_{2} \beta_{2}=1+a^{2} b^{2} c^{2}\left(D_{1} \mathbf{n}, D_{1} n\right) \beta_{1} \beta_{2} .
$$

Substitution of (35) in (32) leads to:

$$
\begin{aligned}
& \frac{\left(D_{1} \boldsymbol{n}, D_{1} \boldsymbol{r}\right)^{2}}{\left(D_{1} \boldsymbol{r}, D_{1} \boldsymbol{r}\right)}+\frac{\left(D_{1} \boldsymbol{n}, D_{1} \boldsymbol{s}\right)^{2}}{\left(D_{1} \boldsymbol{s}, D_{1} \boldsymbol{s}\right)} \\
& =\left(D_{1} \boldsymbol{n}, D_{1} \boldsymbol{n}\right)-\frac{1}{a^{2} b^{2} c^{2} \beta_{1} \beta_{2}} .
\end{aligned}
$$

Because of (24)

$$
a^{2} b^{2} c^{2} \beta_{1} \beta_{2}=a^{2} n_{1}^{2}+b^{2} n_{2}^{2}+c^{2} n_{3}^{2}
$$

holds and with (13) one finally obtains relation (25)

$$
\begin{aligned}
d & =\kappa^{2}\left(\left(D_{1} \boldsymbol{n}, D_{1} \boldsymbol{n}\right)-\frac{\left(D_{1} \boldsymbol{n}, D_{1} \boldsymbol{r}\right)^{2}}{\left(D_{1} \boldsymbol{r}, D_{1} \boldsymbol{r}\right)}-\frac{\left(D_{1} \boldsymbol{n}, D_{1} \boldsymbol{s}\right)^{2}}{\left(D_{1} \boldsymbol{s}, D_{1} \boldsymbol{s}\right)}\right) \\
& =\frac{\kappa^{2}}{a^{2} n_{1}^{2}+b^{2} n_{2}^{2}+c^{2} n_{3}^{2}} .
\end{aligned}
$$

Corollary 3: Under the assumptions of Theorem 1 the area $F$ of the ellipse obtained by the intersection of the ellipsoid (1) and a plane with unit normal vector $\boldsymbol{n}$ and distance $\kappa$ from the origin is given by:

$$
F=\pi\left(1-\frac{\kappa^{2}}{\kappa_{t}^{2}}\right) \frac{a b c}{\kappa_{t}}
$$

with $\kappa_{t}=\sqrt{a^{2} n_{1}^{2}+b^{2} n_{2}^{2}+c^{2} n_{3}^{2}}$.

This is proven by the formula for the area of an ellipse:

$$
F=\pi A B=\pi \sqrt{\frac{1-d}{\beta_{1}}} \sqrt{\frac{1-d}{\beta_{2}}}=\pi \frac{1-d}{\sqrt{\beta_{1} \beta_{2}}}
$$

and by applying (25) and (37). The area of intersection $F$ becomes zero in case $\kappa=\kappa_{t}$ holds; this corresponds to the limiting case, where the cutting plane becomes a tangent plane. This result has been applied in [6].

\section{The Center of the Ellipse}

Substituting $\boldsymbol{q}$ according to (12) in formulars (9) for the 
coordinates $\left(t_{0}, u_{0}\right)$ of the center of the ellipse in the plane spanned by $\boldsymbol{r}$ and $\boldsymbol{s}$ one obtains:

$$
t_{0}=-\kappa \frac{\left(D_{1} \boldsymbol{n}, D_{1} \boldsymbol{r}\right)}{\beta_{1}}-\mu
$$

and

$$
u_{0}=-\kappa \frac{\left(D_{1} \boldsymbol{n}, D_{1} \boldsymbol{s}\right)}{\beta_{2}}-v .
$$

The center $\boldsymbol{m}$ of the ellipse in $R^{3}$ is given by:

$$
\begin{aligned}
\boldsymbol{m} & =\boldsymbol{q}+t_{0} \boldsymbol{r}+u_{0} \boldsymbol{s}=\kappa \boldsymbol{n}+\left(t_{0}+\mu\right) \boldsymbol{r}+\left(u_{0}+v\right) \boldsymbol{s} \\
& =\kappa\left(\boldsymbol{n}-\frac{\left(D_{1} \boldsymbol{n}, D_{1} \boldsymbol{r}\right)}{\beta_{1}} \boldsymbol{r}-\frac{\left(D_{1} \boldsymbol{n}, D_{1} \boldsymbol{s}\right)}{\beta_{2}} \boldsymbol{s}\right) .
\end{aligned}
$$

Theorem 3: Let the assumptions of Theorem 1 be fulfilled. For the center $\boldsymbol{m}$ of the ellipse of intersection in $R^{3}$ holds:

$$
\boldsymbol{m}=\frac{\kappa}{\kappa_{t}^{2}}\left(a^{2} n_{1}, b^{2} n_{2}, c^{2} n_{3}\right)^{\mathrm{T}} .
$$

Proof: With diagonal matrices $D$ from (27) and $\tilde{D}_{1}$ from (22) utilising $\frac{1}{a^{2} b^{2} c^{2}} D^{2}=\tilde{D}_{1}^{2}$ and (37) one obtains a representation of $\boldsymbol{m}$ equivalent to (40):

$$
\boldsymbol{m}=\frac{\kappa}{a^{2} n_{1}^{2}+b^{2} n_{2}^{2}+c^{2} n_{3}^{2}} D^{2} \boldsymbol{n}=\frac{\kappa}{\beta_{1} \beta_{2}} \tilde{D}_{1}^{2} \boldsymbol{n} .
$$

It is sufficient to show that for the difference

$$
\boldsymbol{\Delta}=\boldsymbol{m}-\frac{\kappa}{\beta_{1} \beta_{2}} \tilde{D}_{1}^{2} \boldsymbol{n}
$$

$(\Delta, n)=(\Delta, r)=(\Delta, s)=0$ holds. Thus the coefficients in the expansion of $\boldsymbol{\Delta}$ in $R^{3}$ with respect to the orthonormal basis $[\boldsymbol{n}, \boldsymbol{r}, \boldsymbol{s}]$ are zero, i.e., $\boldsymbol{\Delta}$ is the zero vector.

Applying representation (39) one obtains:

$$
\begin{aligned}
(\boldsymbol{\Delta}, \boldsymbol{n}) & =(\boldsymbol{m}, \boldsymbol{n})-\frac{\kappa}{\beta_{1} \beta_{2}}\left(\tilde{D}_{1}^{2} \boldsymbol{n}, \boldsymbol{n}\right) \\
& =\frac{\kappa}{\beta_{1} \beta_{2}}\left(\beta_{1} \beta_{2}-\left(\tilde{D}_{1} \boldsymbol{n}, \tilde{D}_{1} \boldsymbol{n}\right)\right)=0 .
\end{aligned}
$$

The last expression is zero according to (24). Furthermore one obtains:

$$
\begin{aligned}
(\boldsymbol{\Delta}, \boldsymbol{r}) & =(\boldsymbol{m}, \boldsymbol{r})-\frac{\kappa}{\beta_{1} \beta_{2}}\left(\tilde{D}_{1}^{2} \boldsymbol{n}, \boldsymbol{r}\right) \\
& =-\frac{\kappa}{\beta_{1} \beta_{2}}\left(\left(D_{1} \boldsymbol{n}, D_{1} \boldsymbol{r}\right)\left(D_{1} \boldsymbol{s}, D_{1} \boldsymbol{s}\right)+\left(\tilde{D}_{1} \boldsymbol{n}, \tilde{D}_{1} \boldsymbol{r}\right)\right)
\end{aligned}
$$

and by interchanging the roles of $\boldsymbol{r}$ and $\boldsymbol{s}$ :

$$
\begin{aligned}
(\boldsymbol{\Delta}, \boldsymbol{s}) & =(\boldsymbol{m}, \boldsymbol{s})-\frac{\kappa}{\beta_{1} \beta_{2}}\left(\tilde{D}_{1}^{2} \boldsymbol{n}, \boldsymbol{s}\right) \\
& =-\frac{\kappa}{\beta_{1} \beta_{2}}\left(\left(D_{1} \boldsymbol{n}, D_{1} \mathbf{s}\right)\left(D_{1} \boldsymbol{r}, D_{1} \boldsymbol{r}\right)+\left(\tilde{D}_{1} \boldsymbol{n}, \tilde{D}_{1} \mathbf{s}\right)\right) .
\end{aligned}
$$

Both previous expressions are zero; this follows by applying diagonality condition (7), the identity of Lagrange (14) and Corollary 2:

$$
\begin{aligned}
& \left(D_{1} \boldsymbol{n}, D_{1} \boldsymbol{r}\right)\left(D_{1} \boldsymbol{s}, D_{1} \boldsymbol{s}\right) \\
& =\left(D_{1} \boldsymbol{n}, D_{1} \boldsymbol{r}\right)\left(D_{1} \boldsymbol{s}, D_{1} \boldsymbol{s}\right)-\left(D_{1} \boldsymbol{r}, D_{1} \boldsymbol{s}\right)\left(D_{1} \boldsymbol{s}, D_{1} \boldsymbol{n}\right) \\
& =\left(D_{1} \boldsymbol{r} \times D_{1} \boldsymbol{s}, D_{1} \boldsymbol{n} \times D_{1} \boldsymbol{s}\right)=-\left(\tilde{D}_{1} \boldsymbol{n}, \tilde{D}_{1} \boldsymbol{r}\right) .
\end{aligned}
$$

Interchanging the roles of $\boldsymbol{r}$ and $\boldsymbol{s}$ leads to:

$$
\begin{aligned}
& \left(D_{1} \boldsymbol{n}, D_{1} \boldsymbol{s}\right)\left(D_{1} \boldsymbol{r}, D_{1} \boldsymbol{r}\right) \\
& =\left(D_{1} \boldsymbol{n}, D_{1} \boldsymbol{s}\right)\left(D_{1} \boldsymbol{r}, D_{1} \boldsymbol{r}\right)-\left(D_{1} \boldsymbol{s}, D_{1} \boldsymbol{r}\right)\left(D_{1} \boldsymbol{r}, D_{1} \boldsymbol{n}\right) \\
& =\left(D_{1} \boldsymbol{s} \times D_{1} \boldsymbol{r}, D_{1} \boldsymbol{n} \times D_{1} \boldsymbol{r}\right)=-\left(\tilde{D}_{1} \boldsymbol{n}, \tilde{D}_{1} \boldsymbol{s}\right) .
\end{aligned}
$$

Corollary 4: The apexes of the ellipse of intersection are given by

$$
\boldsymbol{m} \pm A \boldsymbol{r} \text { with } \boldsymbol{m} \pm B \boldsymbol{s},
$$

where $A$ and $B$ are denoting the semi-axes according to $(10)$.

Clearly $\boldsymbol{m} \pm A \boldsymbol{r}$ and $\boldsymbol{m} \pm B \boldsymbol{s}$ are points of the plane cutting the ellipsoid. In order to show that they are belonging to the ellipse of intersection, it has to be verified that they are situated on the ellipsoid, i.e. the following equalities hold:

$$
\begin{aligned}
& \left(D_{1}(\boldsymbol{m} \pm A \boldsymbol{r}), D_{1}(\boldsymbol{m} \pm A \boldsymbol{r})\right)=1 \\
& \left(D_{1}(\boldsymbol{m} \pm B \boldsymbol{s}), D_{1}(\boldsymbol{m} \pm B \boldsymbol{s})\right)=1 .
\end{aligned}
$$

This can be shown using $\boldsymbol{m}$ in the form (39) and employing condition (7).

Corollary 5: $d<1$ holds if and only if $\boldsymbol{m}$ is an interior point of the ellipsoid (1), because of

$$
\left(D_{1} \boldsymbol{m}, D_{1} \boldsymbol{m}\right)=\frac{\kappa^{2}}{\kappa_{t}^{2}}=d .
$$

In the case of $\kappa=\kappa_{t}$, i.e. $d=1$, for the semi-axes (10) of the ellipse of intersection $A=B=0$ follows. The center (40) of the ellipse of intersection becomes a tangent contact point

$$
\boldsymbol{m}=\boldsymbol{m}_{t}=\frac{1}{\kappa_{t}}\left(a^{2} n_{1}, b^{2} n_{2}, c^{2} n_{3}\right)^{\mathrm{T}}
$$

of ellipsoid (1) and a tangent plane with normal vector $\boldsymbol{n}$, since $\left(\boldsymbol{m}_{t}, \boldsymbol{n}\right)=\kappa_{t}$ holds.

Corollary 6: Describing the ellipse of intersection (8) in parametric form 


$$
\begin{aligned}
& t-t_{0}=A \cos \theta \\
& u-u_{0}=B \sin \theta
\end{aligned}
$$

with $\theta \in[0,2 \pi)$, where $A$ and $B$ are denoting its semi-axes according to (10), leads to a representation as a curve in three dimensional space as indicated in [7]

$$
\boldsymbol{x}=\boldsymbol{m}+(A \cos \theta) \boldsymbol{r}+(B \sin \theta) \boldsymbol{s} .
$$

This result may be derived substituting the parameters $t$ and $u$ from the parametric form of the ellipse into Equation (2) of the plane:

$$
\boldsymbol{x}=\boldsymbol{q}+\left(t_{0}+A \cos \theta\right) \boldsymbol{r}+\left(u_{0}+B \sin \theta\right) \boldsymbol{s}
$$

or

$$
\boldsymbol{x}=\boldsymbol{q}+t_{0} \boldsymbol{r}+u_{0} \boldsymbol{s}+(A \cos \theta) \boldsymbol{r}+(B \sin \theta) \boldsymbol{s},
$$

where $\boldsymbol{q}+t_{0} \boldsymbol{r}+u_{0} \boldsymbol{s}$ is equal to the center $\boldsymbol{m}$ of the ellipse as in (39).

\section{Applications}

As indicated in [2], viewing a section through an ellipsoidal eye from a viewpoint normal to the intersection plane and displaying the intersection on that plane along with a projection of the eye structures and isodose lines, radio-therapy treatment of the eye can be planned. For this purpose the line of intersection of ellipsoid (1) and the plane, having the normal vector $(i, j, k)^{\mathrm{T}}$ and containing the point $\boldsymbol{q}=\left(q_{1}, q_{2}, q_{3}\right)^{\mathrm{T}}$, situated in the interior of (1), is determined. The plane has the form:

$$
\left(x-q_{1}\right) i+\left(y-q_{2}\right) j+\left(z-q_{3}\right) k=0
$$

with the unit normal vector:

$$
\boldsymbol{n}=\left(\frac{i}{\sqrt{i^{2}+j^{2}+k^{2}}}, \frac{j}{\sqrt{i^{2}+j^{2}+k^{2}}}, \frac{k}{\sqrt{i^{2}+j^{2}+k^{2}}}\right)^{\mathrm{T}} .
$$

The distance of the plane from the origin is given by:

$$
\kappa=(\boldsymbol{q}, \boldsymbol{n})=\frac{q_{1} i+q_{2} j+q_{3} k}{\sqrt{i^{2}+j^{2}+k^{2}}} .
$$

According to (25) $d$ can be written as:

$$
d=\kappa^{2} \frac{\left(i^{2}+j^{2}+k^{2}\right)}{a^{2} i^{2}+b^{2} j^{2}+c^{2} k^{2}} \text {. }
$$

From (11) it is obvious that $d<1$ holds, as for $\boldsymbol{q}$ as an interior point of the ellipsoid $\left(D_{1} \boldsymbol{q}, D_{1} \boldsymbol{q}\right)<1$ is true. Substituting (18) into (10) the semi-axes of the ellipse, the line of intersection of ellipsoid and plane, are given by

$$
A=\sqrt{\frac{1-d}{\beta_{1}}} \text { and } B=\sqrt{\frac{1-d}{\beta_{2}}}
$$

where $\beta_{1}, \beta_{2}$ are solutions of Equation (19):

$$
\begin{aligned}
& \left(i^{2}+j^{2}+k^{2}\right) \beta^{2} \\
& -\left[i^{2}\left(\frac{1}{b^{2}}+\frac{1}{c^{2}}\right)+j^{2}\left(\frac{1}{a^{2}}+\frac{1}{c^{2}}\right)+k^{2}\left(\frac{1}{a^{2}}+\frac{1}{b^{2}}\right)\right] \beta \\
& +\frac{i^{2}}{b^{2} c^{2}}+\frac{j^{2}}{a^{2} c^{2}}+\frac{k^{2}}{a^{2} b^{2}}=0 .
\end{aligned}
$$

With Theorem 3 one obtains by substituting $\boldsymbol{n}$ and $\kappa$ from (42) and (43) the formular for the center $\boldsymbol{m}$ of the ellipse given by:

$$
\boldsymbol{m}=\frac{\kappa\left(i^{2}+j^{2}+k^{2}\right)}{\left(a^{2} i^{2}+b^{2} j^{2}+c^{2} k^{2}\right) \sqrt{i^{2}+j^{2}+k^{2}}}\left(a^{2} i, b^{2} j, c^{2} k\right)^{\mathrm{T}} .
$$

Instead of calculating $\beta_{1}$ and $\beta_{2}$ as solutions of (46) they may be obtained alternatively using the procedure described in $\S 2$. Starting with an arbitrary unit vector $\boldsymbol{r}$ orthogonal to the unit normal vector $\boldsymbol{n}$ given in (42), e.g.

$$
\boldsymbol{r}=\left(\frac{j}{\sqrt{i^{2}+j^{2}}}, \frac{-i}{\sqrt{i^{2}+j^{2}}}, 0\right)^{\mathrm{T}}
$$

calculating $\boldsymbol{s}$ to be orthogonal to both according to $\boldsymbol{s}=\boldsymbol{n} \times \boldsymbol{r}$ and, in case $\left(D_{1} \boldsymbol{r}, D_{1} \boldsymbol{s}\right) \neq 0$, perform a rotation with angle $\omega$ as described in $\S 2$, yielding new vectors $\tilde{\boldsymbol{r}}$ and $\tilde{\boldsymbol{s}}$, which are plugged into (18).

A Mathematica program containing both ways of computation of $\beta_{1}$ and $\beta_{2}$ may be obtained from the author upon request.

In the first special case of a plane containing the origin (see e.g. [1]), i.e. $\boldsymbol{q}$ is the zero vector, it follows by (43), (44) and (47) that $\kappa=0, d=0$ and $\boldsymbol{m}$ is the zero vector also. Furthermore the semi-axes of the ellipse in (45) reduce to

$$
A=\frac{1}{\sqrt{\beta_{1}}} \text { and } B=\frac{1}{\sqrt{\beta_{2}}}
$$

and from (9) $t_{0}=u_{0}=0$ holds. Thus Equation (8) of the line of intersection reduces to

$$
\beta_{1} t^{2}+\beta_{2} u^{2}=1
$$

A second special case, where $\boldsymbol{q}=(i, j, k)^{\mathrm{T}}$ holds, was treated in [2]. Then the above formulas (43), (44) and (47) reduce to:

$$
\kappa=\sqrt{i^{2}+j^{2}+k^{2}}, \quad d=\frac{\left(i^{2}+j^{2}+k^{2}\right)^{2}}{a^{2} i^{2}+b^{2} j^{2}+c^{2} k^{2}}
$$

and

$$
\boldsymbol{m}=\frac{i^{2}+j^{2}+k^{2}}{\left(a^{2} i^{2}+b^{2} j^{2}+c^{2} k^{2}\right)}\left(a^{2} i, b^{2} j, c^{2} k\right)^{\mathrm{T}} .
$$


Because of $\boldsymbol{q}=\kappa \boldsymbol{n}$ in (12) $\mu=v=0$ holds and (38) reduces to

$$
t_{0}=-\kappa \frac{\left(D_{1} \boldsymbol{n}, D_{1} \boldsymbol{r}\right)}{\beta_{1}} \text { and } u_{0}=-\kappa \frac{\left(D_{1} \boldsymbol{n}, D_{1} s\right)}{\beta_{2}},
$$

where $\beta_{1}$ and $\beta_{2}$ are solutions of the quadratic Equation (46) and vectors $r$ and $s$ have to be determined as described above according to the procedure shown in $\S 2$. Thus Equation (8) of the line of intersection turns into:

$$
\beta_{1} \frac{\left(t-t_{0}\right)^{2}}{1-d}+\beta_{2} \frac{\left(u-u_{0}\right)^{2}}{1-d}=1
$$

\section{Conclusion}

The intention of this paper was, to give an elementary closed form solution to the general problem of the intersection of an ellipsoid and a plane.

\section{REFERENCES}

[1] The Math Forum, "Intersection of Ellipsoid and Plane," 2007. http://mathforum.org/library/drmath/view/71275.html

[2] The Math Forum, "Ellipsoid and Plane Intersection Equation," 2000.

http://mathforum.org/library/drmath/view/51781.html

[3] The Math Forum, "Intersection of Hyperplane and an Ellipsoid," 2007. http://mathforum.org/library/drmath/view/72315.html

[4] A. Korn and M. Korn, "Mathematical Handbook for Scientists and Engineers," Mc Graw-Hill Book Company, Inc., New York, Toronto, London, 1961.

[5] C. C. Ferguson, "Intersections of Ellipsoids and Planes of Arbitrary Orientation and Position," Mathematical Geology, Vol. 11, No. 3, 1979, pp. 329-336. doi:10.1007/BF01034997

[6] M. P. Verma and J. C. Upadhyaya, "On the Electron-Ion Interaction in Hexagonal and Tetragonal Metals," Journal of Physics F: Metal Physics, Vol. 1, No. 5, 1971, pp. 618620. doi:10.1088/0305-4608/1/5/315

[7] The Math Forum, "Equation of an Ellipse in 3-Space," 2003.

http://mathforum.org/library/drmath/view/63373.html 